\title{
Dinámica temporal de la disponibilidad de agua y microclima en aguadas del biotopo protegido Dos Lagunas, Petén, Guatemala
}

\author{
Temporal dynamics of water availability and microclimate in seasonal water ponds in the \\ Dos Lagunas Biotopo Protegido, Peten, Guatemala \\ Manolo J. García*, Andrea L. Aguilera, Gerber D. Guzmán-Flores, Dana I. Rodríguez, Vivian R. González \\ Centro de Estudios Conservacionistas, Facultad de Ciencias Químicas y Farmacia, \\ Universidad de San Carlos de Guatemala, Guatemala.
}

*Autor al que se dirige la correspondencia: garcia.manolo@usac.edu.gt

Recibido: 29 de marzo 2017 / 1era. Revisión: 06 de marzo 2018 / 1era. Revisión: 27 de junio 2018 / Aceptado: 28 de agosto 2018

\section{Resumen}

- $\mathrm{n}$ el territorio de la Selva Maya se han identificado a los cuerpos de agua estacionales denominados localmente - aguadas, como un recurso crítico para la supervivencia de especies de fauna silvestre, ya que son su principal fuente de agua durante la temporada seca. En el presente estudio se registró en cinco aguadas, la dinámica temporal de la acumulación de agua empleando una cámara automática, así como la temperatura y la humedad relativa utilizando sensores automáticos a lo largo de abril a agosto del 2016. En cuanto a la disponibilidad de agua, esta fue disminuyendo hasta agotarse al final de la temporada seca en el mes de abril, y fue aumentando al inicio de la temporada lluviosa en junio. Para la temporada seca se registró una temperatura promedio (desviación estándar) de $27.65(4.52)^{\circ} \mathrm{C}$ y una humedad relativa de $71.30(19.00) \%$, mientras que para la temporada lluviosa la temperatura promedio fue de $26.09(3.21){ }^{\circ} \mathrm{C}$ y humedad relativa promedio de 86.16 (14.15) \%. Sin embargo no se encontró una diferencia significativa entre los valores registrados en las "distintas" aguadas. Se encontró una correlación significativa entre los valores registrados en las aguadas con los registrados por la estación meteorológica automática (EMA) Calakmul II, por lo que para estudios futuros es posible utilizar los valores de la EMA para representar la dinámica en las aguadas. Entre las estrategias para reducir los potenciales efectos del cambio climático para la fauna es la conservación de las aguadas manteniendo la conectividad entre las mismas.

Palabras claves: temperatura ambiental, humedad relativa, diversidad biológica, recursos hídricos, Reserva de Biosfera Maya

\begin{abstract}
I $\mathrm{n}$ the Maya Forest, the seasonal water ponds locally known as aguadas have been identified as a critical resource for the survival of wild species, as they are the main source of fresh water during the dry season. In the present study, in five aguadas, we recorded the availability of water using a camera-trap, as well as the temperature and relative humidity using data loggers, from April to August 2016. Regarding water availability, the water quantity was diminishing at the end of the dry season in April until it ran out, and increased as the rainy season started on June. For the dry season we recorded an average temperature (standard deviation) of $27.65(4.52){ }^{\circ} \mathrm{C}$ and average relative humidity of $71.30(19.00) \%$, and on the rainy season, the average temperature was $26.09(3.21){ }^{\circ} \mathrm{C}$ and average relative humidity of $86.16(14.15) \%$. Nevertheless we didn't found a significant difference between the values recorded at the aguadas. We founded a significant correlation between the values recorded at the aguadas and the Automatic Meteorological Station (AME) Calakmul II, so for future research the AME values can be used to represent the dynamics at the aguadas. Among the strategies to reduce the potential impacts of Climate Change on animal species is the conservation of aguadas, maintaining the connectivity within them.
\end{abstract}

Keywords: Environmental temperature, relative humidity, biological diversity, hydrological resources, Maya Biosphere Reserve 


\section{Introducción}

El territorio denominado Selva Maya, el cual abarca áreas de México, Guatemala y Belice, ha sido identificado como un centro de alta diversidad biológica en la región mesoamericana, que a su vez está amenazado como consecuencia de las elevadas tasas de deforestación en años recientes, así como por los impactos potenciales del cambio climático (Anderson et al., 2008; Hansen et al., 2013; Hodgon, Hughell, Ramos, \& McNab, 2015). En este territorio, de manera general, se presenta un clima de tipo tropical cálido y húmedo, con temperaturas medias entre los 22 y $29^{\circ} \mathrm{C}$ y precipitaciones entre los 1,200 y 2,000 mm anuales, sin una estacionalidad bien definida (Araujo, 2014; Consejo Nacional de Áreas Protegidas [Conap], 2015). A una menor escala, existe un gradiente de humedad y temperatura de norte a sur y de este a oeste, ocasionando que el noreste es más seco y cálido en comparación con el suroeste (Conap, 2015; Instituto Nacional de Ecología [INE], 2000). El área es predominantemente plana (plataforma sedimentaria de Yucatán) con algunos sistemas de serranías hacia el sur, correspondientes a los cinturones plegados de Chiapas y Lacandón (sistemas montañosos del norte de Centroamérica), en un suelo de tipo cárstico (Conap, 2015; INE, 2000). Las características propias del suelo, hace que la mayor parte del drenaje sea subterráneo, con algunos cuerpos de agua dulce que incluyen lagos, lagunas, ríos permanentes e intermitentes y otros tipos de humedales, siendo un elemento sobresaliente las aguadas, las cuales consisten en zonas que almacenan el agua de lluvia, y pueden variar desde $236 \mathrm{~m}^{2}$ hasta varias hectáreas (Araujo, 2014; Conap, 2015; González, 2015; Reyna, O'Farril, Simá, Andrade, Padilla, \& Sosa, 2010). Las aguadas suelen presentarse en bajas densidades, ya que se encuentran dispersas en el paisaje (González, 2015; Reyna et al., 2010). Dado que las aguadas, son formadas por la acumulación del agua de lluvia, presentan una dinámica asociada a las temporadas seca y lluviosa, en la cual algunas aguadas pueden secarse por completo durante la época seca, mientras que otras permanecen a lo largo del año (González, 2015).

Debido a la poca disponibilidad de agua superficial en la región, las aguadas son una de las principales fuentes de agua para mantener a la fauna silvestre durante la época seca (Reyna et al., 2010; Simá, Reyna, $\&$ Retana, 2008). Se ha documentado que más de 20 especies de vertebrados visitan las aguadas, incluyendo especies amenazadas y de importancia económica y cultural, entre las que se incluyen aves como Aramides albiventris Lawrence, 1868 (gallinula), Crax rubra Linnaeus, 1758 (faisán), Meleagris ocellata Cuvier, 1820 (pavo ocelado), así como especies de mamíferos incluyendo Tapirus bairdii Gill, 1865 (danto o tapir), Panthera onca Linnaeus, 1758 (jaguar), Tayassu pecari Link, 1795 (jabalí de labio blanco), Puma concolor Linnaeus, 1771 (puma), Leopardus pardalis Linnaeus, 1758 (ocelote), entre otras (González, 2015; Simá et al., 2008; Ruano et al., 2009). Dos de las especies de mamíferos que presentan una alta dependencia de las aguadas son T. bairdii y T. pecari, por lo que las aguadas son vitales para su supervivencia (Moreira-Ramírez et al., 2016; Pérez-Cortez, Enríquez, Sima-Panti, Reyna-Hurtado, \& Naranjo, 2012; Ruano et al., 2009; Sandoval-Serés, Reyna-Hurtado, Briceño-Méndez, \& de la Cerda-Vega, 2016).

Dada la relevancia que tienen las aguadas para la diversidad biológica, el objetivo de este estudio fue documentar la dinámica temporal de acumulación de agua, temperatura y humedad relativa en cinco aguadas del Biotopo Protegido Dos Lagunas (BPDL), con el fin de conocer la dinámica hidrológica de estos importantes humedales, y así generar insumos que permitan el desarrollo de estrategias de adaptación y mitigación de los efectos del cambio climático para la fauna silvestre en la región de la Selva Maya.

\section{Materiales y métodos}

\section{Área de estudio}

La etapa de campo se realizó en cinco aguadas ubicadas en el BPDL, zona núcleo de la RBM, localizado en el límite norte del municipio de Flores en el departamento de Petén. El biotopo colinda al norte con la Reserva de Biosfera Calakmul (RBC) en Quintana Roo, México y con el Parque Nacional Mirador Río Azul en sus límites sur, este y oeste, contando con una extensión aproximada de 30,710 ha $\left(307.19 \mathrm{~km}^{2}\right)$ (Figura 1) (Conap, Dirección General de Patrimonio Cultural y Natural [DGPCyN], \& Centro de Estudios Conservacionistas [Cecon], 2010). En cuanto al clima, la temperatura promedio varía entre 21 y $30^{\circ} \mathrm{C}$, la precipitación promedio es de $1,136 \mathrm{~mm} /$ año y se presentan variaciones estacionales con una temporada seca de febrero a mayo y otra lluviosa de junio a diciembre (Conap et al., 2010). Las aguadas incluidas en este estudio fueron las conocidas localmente como Monifatos, El Trampole, El Tintal, Poza Maya y otra sin nombre 


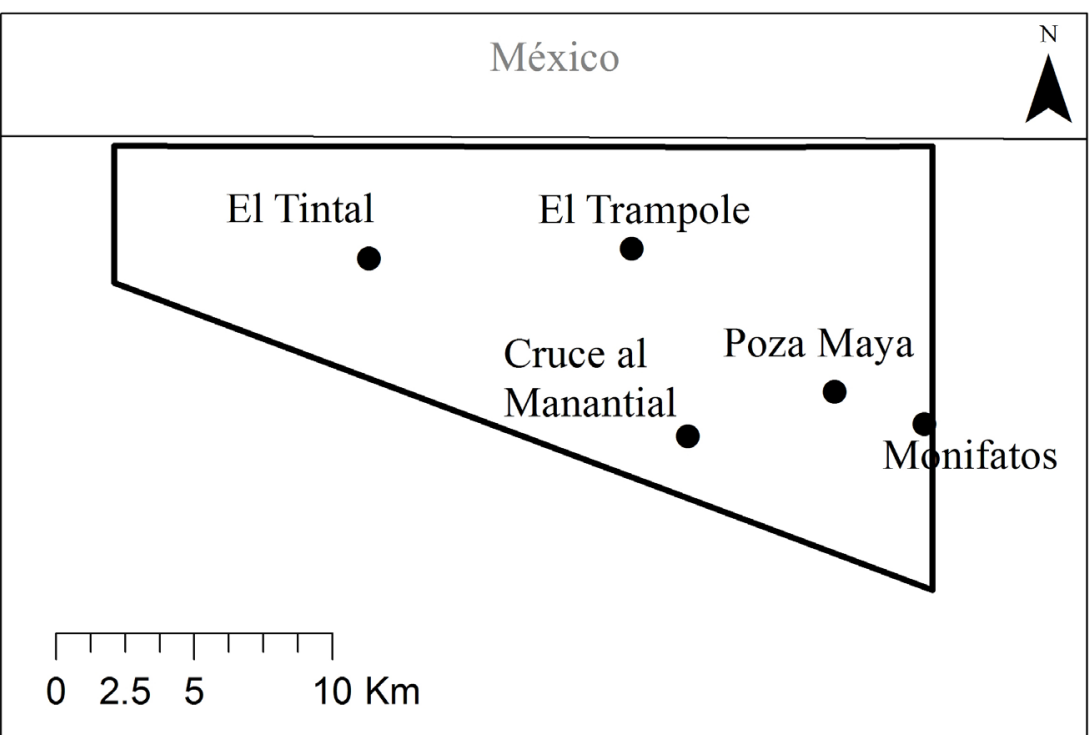

Polígono del Biotopo Protegido Naachtún Dos Lagunas y la ubicación de las aguadas en estudio

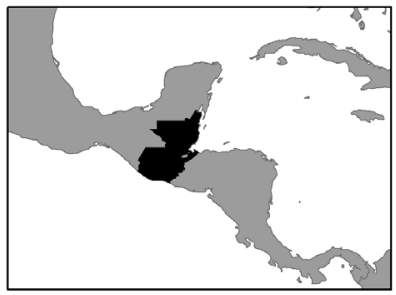

Ubicación de Guatemala en Centroamérica

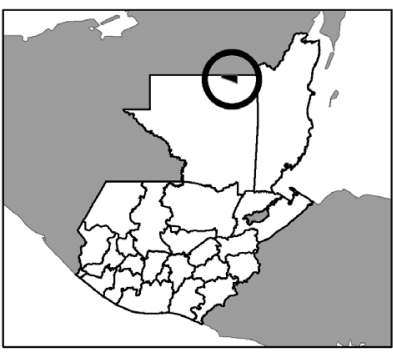

Ubicación del Biotopo en el Departamento de Petén

Figura 1. Ubicación del Biotopo Protegido Dos Lagunas, Petén, Guatemala.

conocido ubicada en el camino hacia el campamento chiclero El Manantial, a la cual se le denominó Cruce al Manantial. El estudio se realizó durante los meses de abril a agosto del 2016, con el fin de registrar la dinámica de la disponibilidad de agua, temperatura y humedad relativa en las aguadas durante el cambio de la temporada seca a la lluviosa.

\section{Registro de la disponibilidad de agua en aguadas}

Se realizó un registro fotográfico de la disponibilidad de agua en cada una de las aguadas del estudio, utilizando cámaras automáticas o cámaras-trampa marca Bushnell modelo Trophycam 14MP Intrussor No Glow (Bushnell outdoor products, Overland Park, Estados Unidos). En cada una de las aguadas, en un árbol ubicado en el margen, se instaló una cámara-trampa orientada hacia el centro del espejo de agua a una altura aproximada de $1.7 \mathrm{~m}$. Con una resolución de 14 megapixeles, una foto por evento de captura y la función field scan (barrido del campo visual) activada para tomar una foto de manera automática a cada hora en punto entre las 05:00 y 18:00 h. Las imágenes obtenidas fueron examinadas con el fin de determinar para cada aguada, la hora del día en que era posible apreciar con mayor claridad el contenido de agua acumulada. Los horarios seleccionados para las aguadas de estudio fueron: Monifatos 17:00 h, Trampole 06:00 h, El Tintal 09:00 h, Poza Maya 07:00 h y Cruce al Manantial 14:00 h.

Con el fin de contar con una referencia para la estimación de la disponibilidad de agua, se colocó un lazo previamente identificado con marcas cada metro desde el centro de la aguada hacia la cámara, activando la cámara de manera manual para que tomara fotografías. Posteriormente, se realizó una compilación de todas las fotos de cada aguada a la hora seleccionada, las cuales fueron comparadas con la referencia para estimar el radio del espejo de agua para cada día, como una referencia de la cantidad de agua acumulada.

\section{Registro de variables microclimáticas}

El registro de la temperatura y humedad relativa de cada aguada se realizó utilizando un sensor automático o data logger marca Onset modelo HOBOS Pro v2 U23-001 (Onset Computer Corporation, Bourne, Estados Unidos), instalado a una altura de $1.7 \mathrm{~m}$ en un árbol cercano a la cámara automática. Para facilitar la recuperación de los dispositivos en el campo se utilizó cinta forestal para identificar los árboles donde 
se instaló el equipo y se tomaron las coordenadas de los mismos utilizando un GPS. Los sensores fueron programados para activarse de manera simultánea y registrar las variables cada hora. Los datos colectados por los sensores automáticos fueron descargados en archivos de texto utilizando el programa HOBOware Pro v. 3.5.0 (Onset Computer Corporation 2002-2013, Bourne, Estados Unidos) y posteriormente exportados a hojas de cálculo electrónicas para la estimación de los promedios semanales.

Se realizó un análisis de varianzas (Andeva) para comparar los datos de temperatura registrados en las aguadas para determinar si existen diferencias significativas entre las mismas.

\section{Correlación entre los datos de temperatura y humedad en aguadas y la Estación Meteorológi- ca Automática (EMA) Calakmul II}

El Servicio Meteorológico Nacional de la Comisión Nacional del Agua (Conagua) de México, posee un sistema de estaciones meteorológicas automáticas (EMAs) para el registro constante de variables climáticas en distintos sitios de México, incluyendo dos estaciones en la RBC. Debido al costo de monitorear las variables microclimáticas directamente en las aguadas, se quiso conocer si los datos de temperatura y humedad colectados por las EMAs poseen una correlación significativa con los que fueron colectados en las aguadas, $y$ así poder utilizar este recurso en análisis y monitoreo de aguadas en el futuro, sin necesidad de instalar sensores en las aguadas. Se realizó un análisis de correlación de Pearson entre los promedios diarios de temperatura y humedad relativa registrados en las aguadas con los datos registrados por la EMA Calakmull II ubicada al norte del BPDL.

\section{Resultados}

\section{Registro de la disponibilidad de agua en aguadas}

Se obtuvieron 7,620 fotografías de las cuales se seleccionaron 635 para ser incluidas en el procesamiento. A partir de esta información se generó una base de datos con 635 registros de los radios diarios estimados para las cinco aguadas de estudio, de los cuales corresponden a El Tintal con 139 días, Monifatos con 146, Poza Maya con 148, Trampole con 127 y Cruce al Manantial con 75. Con estos datos se estimaron los promedios semanales de la disponibilidad de agua paras las aguadas El Tintal, Los Monifatos y Poza Maya. No fue posible estimar el radio para la aguada Trampole debido al crecimiento de la vegetación lo que impidió la visibilidad del espejo de agua y en el caso de la aguada Cruce al Manantial la cámara presentó desperfectos por lo que no se obtuvieron fotografías para todo el período de estudio. De las aguadas que fue posible estimar el radio, las tres se secaron a finales del mes de abril (alrededor de las semanas 16 y 17) y permanecieron totalmente secas durante 5 semanas (de la semana 18 a la 22), hasta que comenzaron a llenarse nuevamente a inicios de la temporada lluviosa en el mes de junio (Figura 2).

\section{Registro de variables microclimáticas}

Con respecto a las variables climáticas, se obtuvieron 16,248 mediciones que corresponden a 2,520 para el Cruce al Manantial y 3,432 para cada una de las otras aguadas. Los valores registrados de ambas variables son similares para las cinco aguadas a lo largo del período de estudio, tanto en la dinámica diaria como entre semanas y temporadas (Tabla 1).

Con respecto a la dinámica diaria de la temperatura y la humedad relativa, a partir del total de datos registrados por los sensores, puede evidenciarse que de manera general que en las aguadas existe un aumento gradual en la temperatura a partir de la hora más fresca entre las 05:00 y 06:00 h, alcanzando su máximo entre las 12:00 y 14:00 h, y posteriormente vuelve a descender gradualmente hasta la hora más fresca (Figura 3). La humedad relativa presentó un patrón inverso, en el cual entre las 5:00 y 7:00 h se alcanza la mayor humedad relativa, la cual desciende gradualmente alcanzando su mínimo entre las 13:00 y 15:00 h para aumentar nuevamente (Figura 4).

En cuanto a los patrones a lo largo de las semanas, con respecto a la temperatura promedio se presentan fluctuaciones entre las semanas, con una tendencia al incremento durante la época seca en los meses de abril y mayo, y un decremento al inicio de la temporada lluviosa en el mes de junio (Figura 5). Durante las semanas 21 y 22 en el mes de mayo se alcanzaron las máximas temperaturas promedio (desviación estándar) de 29.40 (4.34) y $29.02(4.07){ }^{\circ} \mathrm{C}$, respectivamente, mientras que la más baja, 24.38 (2.53) se registró en la semana 31 en el mes de julio. A pesar que los valores de temperatura son muy similares en las aguadas, puede notarse que durante las semanas más cálidas ( 21 y 22) del período 

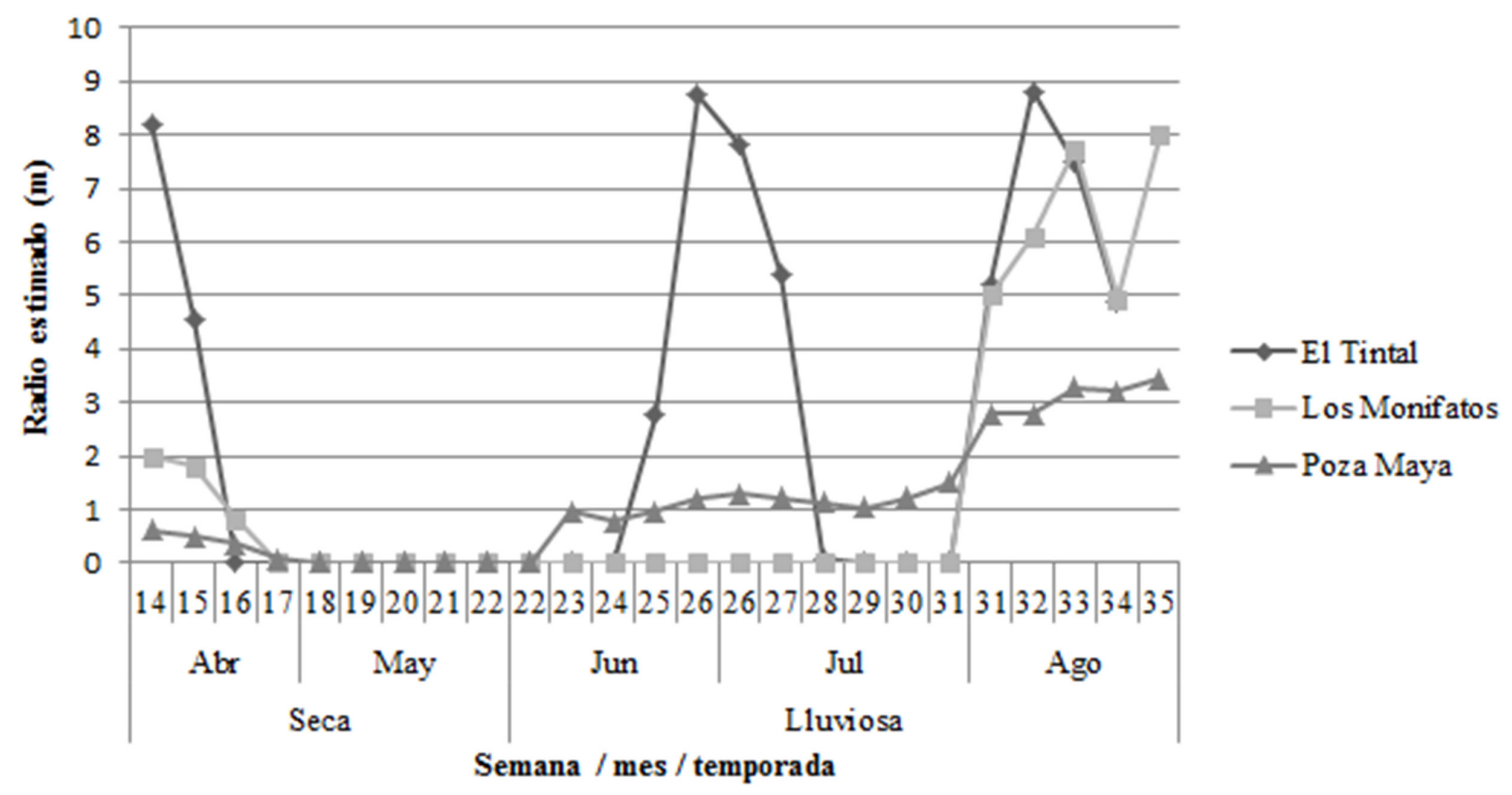

Figura 2. Promedio semanal de la estimación del radio por aguada.

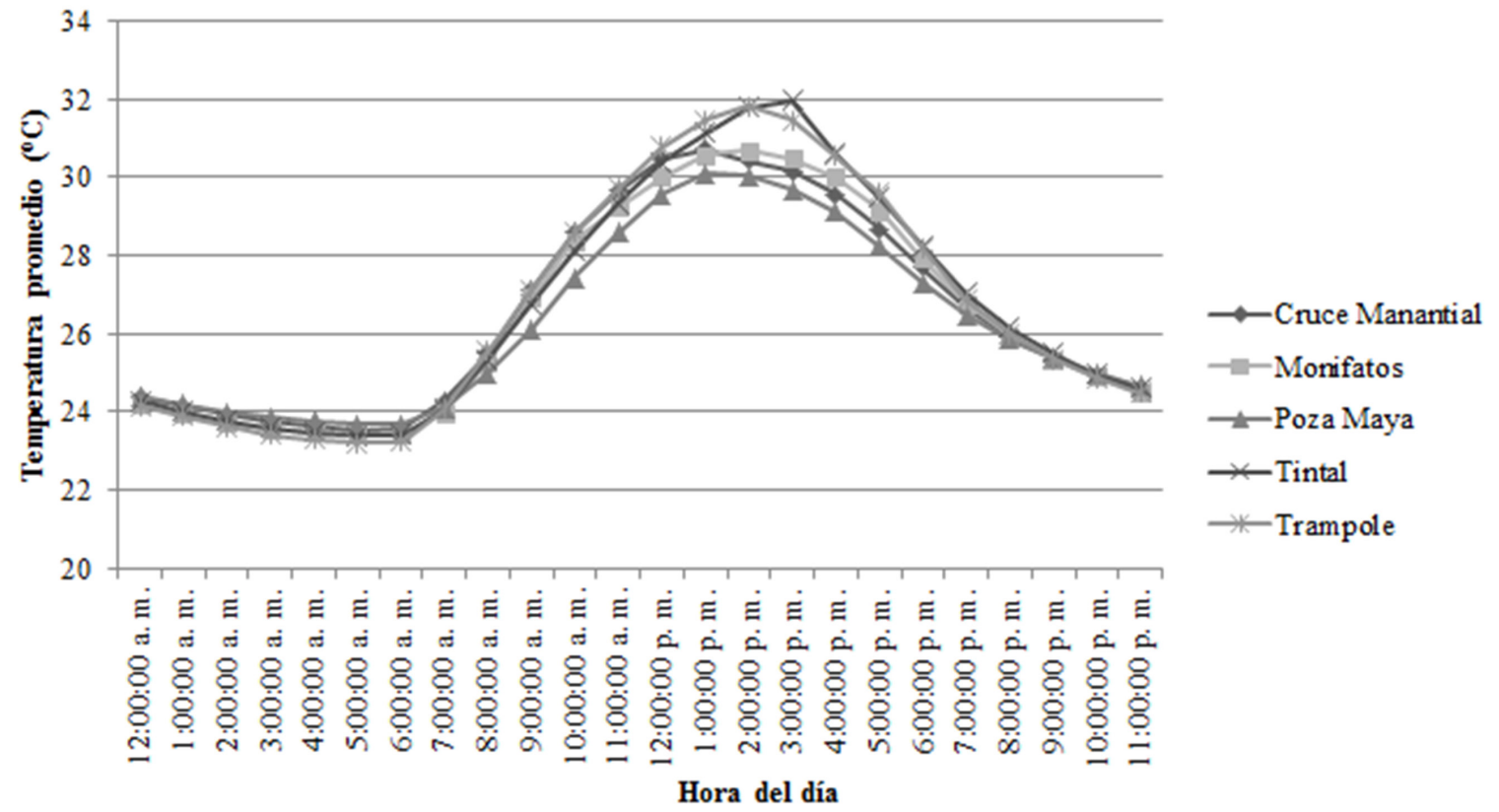

Figura 3. Dinámica diaria de temperatura en cinco aguadas para el periodo del estudio. 
Tabla 1

Valores promedio y desviación estándar de temperatura y humedad relativa por aguada

\begin{tabular}{|c|c|c|c|c|c|}
\hline \multirow{3}{*}{ Variable } & \multirow{3}{*}{ Aguada } & \multicolumn{4}{|c|}{ Temporada } \\
\hline & & \multicolumn{2}{|r|}{ Seca } & \multicolumn{2}{|c|}{ Lluviosa } \\
\hline & & Promedio & Desviación estándar & Promedio & $\begin{array}{c}\text { Desviación } \\
\text { estándar }\end{array}$ \\
\hline \multirow[t]{5}{*}{ Temperatura } & Cruce Manantial & 29.3 & $(4.4)$ & 26.0 & $(3.2)$ \\
\hline & Monifatos & 27.4 & $(4.1)$ & 25.9 & $(2.9)$ \\
\hline & Poza Maya & 27.3 & $(3.8)$ & 25.6 & $(2.5)$ \\
\hline & Tintal & 27.8 & $(4.5)$ & 26.1 & $(3.3)$ \\
\hline & Trampole & 27.8 & $(4.8)$ & 26.1 & $(3.3)$ \\
\hline \multirow[t]{5}{*}{$\begin{array}{l}\text { Humedad } \\
\text { relativa }\end{array}$} & Cruce Manantial & 69.0 & (19.9) & 86.9 & $(14.1)$ \\
\hline & Monifatos & 72.1 & $(17.9)$ & 87.1 & $(13.6)$ \\
\hline & Poza Maya & 73.2 & $(17.0)$ & 90.2 & (11.6) \\
\hline & Tintal & 69.7 & $(18.3)$ & 85.3 & $(14.3)$ \\
\hline & Trampole & 70.8 & $(19.2)$ & 86.8 & $(14.2)$ \\
\hline
\end{tabular}

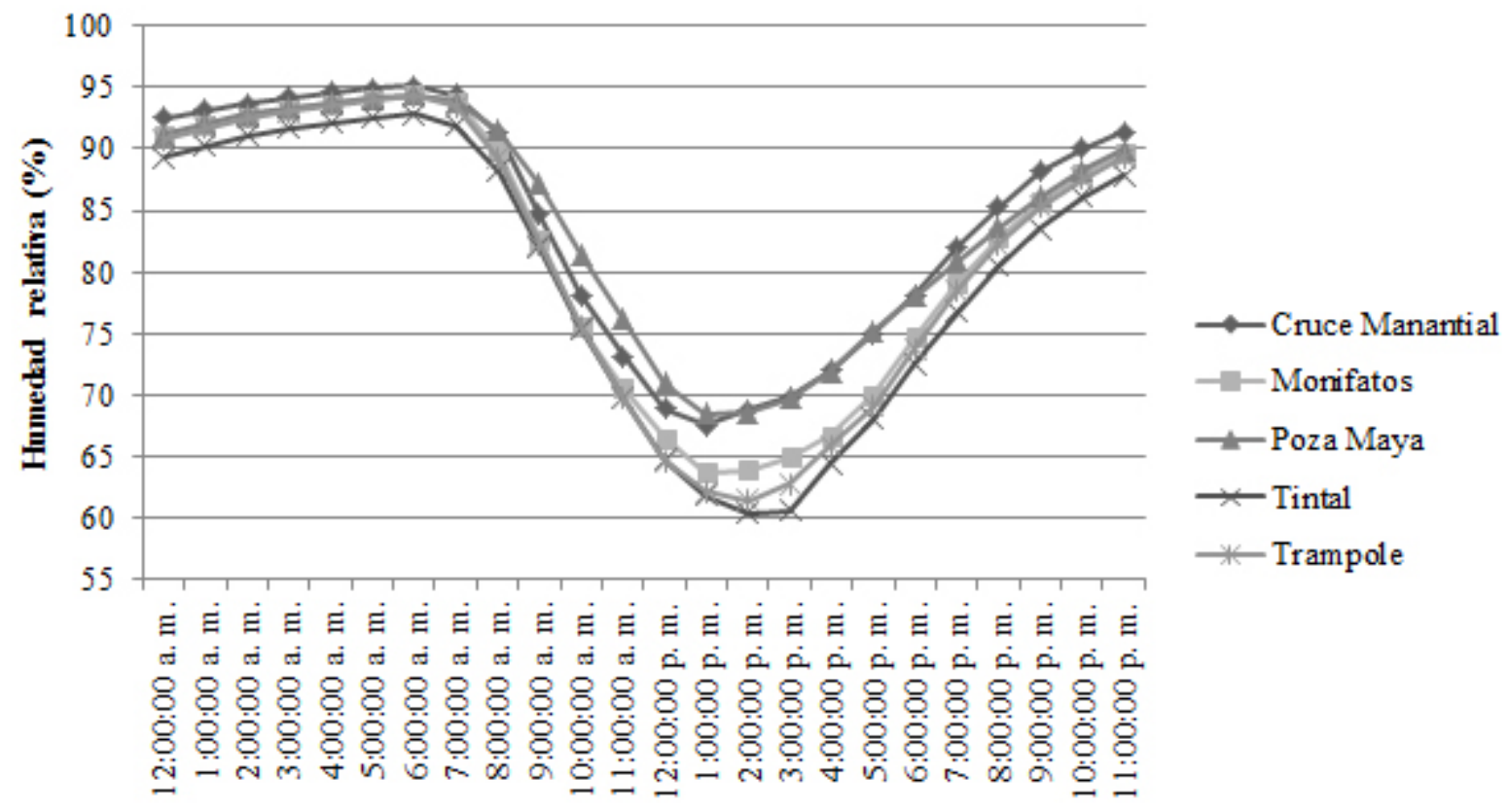

Hora del día

Figura 4. Dinámica diaria de humedad relativa en cinco aguadas para el periodo del estudio. 


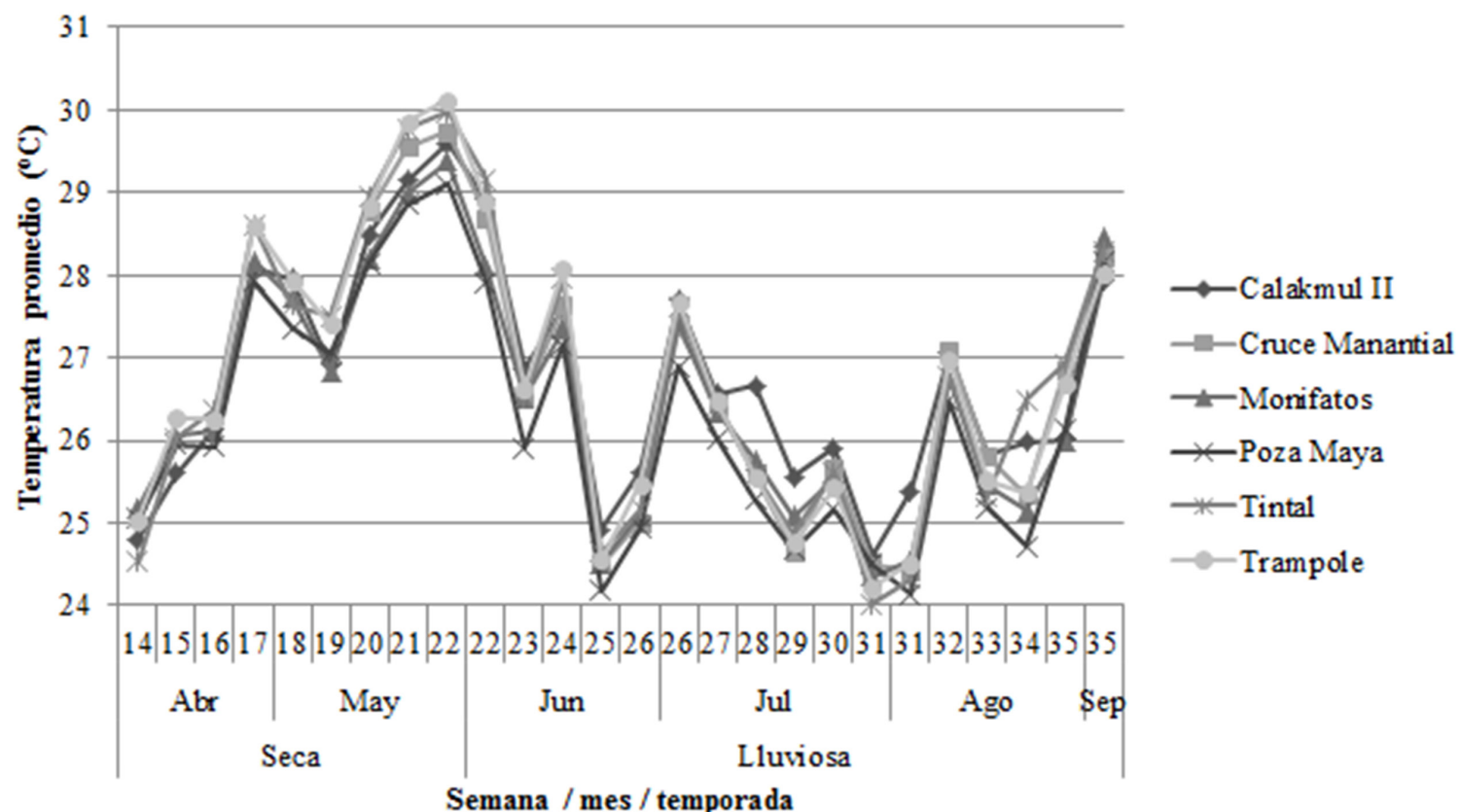

Figura 5. Promedio semanal de la temperatura $\left({ }^{\circ} \mathrm{C}\right)$ en cada una de las aguadas en estudio y la estación meteorológica automática Calakmul II. Nota: Abr=abril, May=mayo, Jun=junio, Jul=julio, Ago=agosto, Sep=septiembre..

del estudio, las aguadas Cruce al Manantial, El Tintal y Trampole alcanzaron temperaturas mayores con respecto a las aguadas Los Monifatos y Poza Maya, siendo esta última la que presenta en la mayoría de las semanas los promedios menores de temperatura. Así mismo, puede notarse que la temperatura ambiental registrada por la EMA Calakmul II es ligeramente mayor a la de las aguadas durante las semanas 28 a la 30 en el mes de junio. La mayor variación de los valores entre las aguadas ocurrió en la semana 34 en el mes de agosto.

Con respecto a la humedad relativa puede observarse un patrón similar para todas las aguadas, sin embargo es notorio que la Poza Maya presenta valores promedio mayores que las otras aguadas, especialmente durante la temporada lluviosa, a partir de la semana 25 (Figura 6). Durante las semanas de la 18 a la 22, cuando se presentaron los valores más bajos de humedad relativa, la aguada El Tintal presentó los valores promedio más bajos de humedad relativa. Así mismo, la humedad relativa ambiental registrada por la EMA Calakmul II presenta valores promedio menores que las aguadas, de la semana 27 a la 33 correspondientes a los meses de julio y agosto.
A pesar de que se observan algunas diferencias entre los valores de temperatura y humedad relativa en las aguadas, en el Andeva se obtuvo un valor F de $0.3869(\mathrm{P}=0.817563)$ con lo que se determinó que no existen diferencias significativas entre las aguadas. (Tabla 2)

\section{Correlación entre temperatura y humedad re- lativa en aguadas y la EMA}

Se estimaron los coeficientes de correlación entre los valores promedio diarios de temperatura de la EMA Calakmul II y los valores en las aguadas (Tabla 3). El promedio (desviación estándar) de las correlaciones entre la EMA y los sensores automáticos fue de 0.8638 (0.02). Para la humedad relativa se presentó una tendencia similar, con un promedio (desviación estándar) para las correlaciones de $0.8425(0.02)$. 


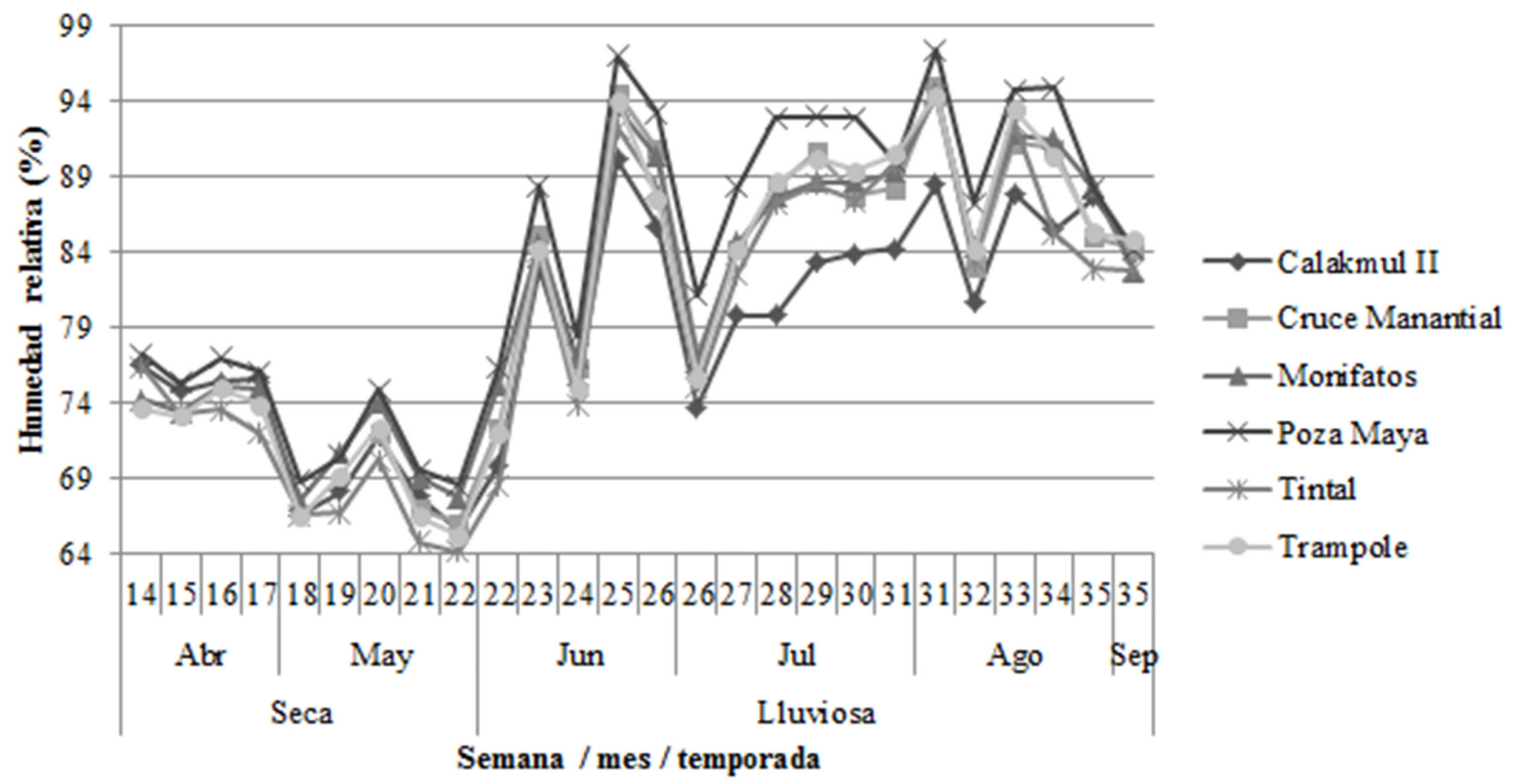

Figura 6. Promedio semanal de la humedad relativa (\%) en cada una de las aguadas en estudio y la estación meteorológica automática Calakmul II. Nota: Abr=abril, May=mayo, Jun=junio, Jul=julio, Ago=agosto, Sep=septiembre.

Tabla 2

Valores del análisis de varianza (Andeva) para los valores de temperatura promedio semanal para las aguadas del estudio

\begin{tabular}{lrrrrr}
\hline \multicolumn{1}{c}{ Fuente } & Suma de cuadrados & Grados de libertad & Cuadrado medio & F & \multicolumn{1}{c}{ P } \\
\hline Entre grupos & 3.4511 & 4 & 0.8628 & 0.3869 & 0.817563 \\
Error & 220.7485 & 99 & 2.2298 & & \\
Total & 224.1996 & 103 & & & \\
\hline
\end{tabular}

Tabla 3

Coeficientes de correlación entre la temperatura registrada por la EMA Calakmul II y los data loggers en las aguadas

\begin{tabular}{crrrrr}
\hline \multicolumn{1}{c}{ Aguada } & $\begin{array}{c}\text { Cruce } \\
\text { Manantial }\end{array}$ & \multicolumn{1}{c}{ Monifatos } & Poza Maya & Tintal & Trampole \\
\hline $\begin{array}{l}\text { Coeficiente de correlación } \\
\text { con la EMA Calakmul II }\end{array}$ & 0.8676759 & 0.8681748 & 0.8350361 & 0.868933 & 0.8792934 \\
\hline
\end{tabular}




\section{Discusión}

\section{Registro de la disponibilidad de agua en aguadas}

En los cuerpos de agua estacionales someros, como las aguadas, el patrón temporal de la acumulación de agua (hidrorégimen) y su duración en las mismas (hidroperíodo) son determinadas por el balance hídrico entre el abastecimiento y la pérdida del líquido. Los componentes principales de este balance son la precipitación, los flujos superficiales y subterráneos y la evapotranspiración, los cuales interactúan en una combinación particular en función a las particularidades del cuerpo de agua (Leibowitz \& Brooks, 2007; Winter, Rosenberry, Buso, \& Merk, 2001). Estudios demuestran cómo en un cuerpo de agua somero, el hidroperiodo refleja en primera instancia la variación en el clima, especialmente de la precipitación como fuente de recarga hídrica (Brooks, 2004; Winter et al., 2001; Zedler, 1987).

Dada la naturaleza de las aguadas, el aumento del radio de su espejo de agua corresponde con eventos climáticos de precipitación (Reyna et al., 2010). En la Poza Maya y El Tintal este aumento es evidente al inicio de la temporada lluviosa en el mes de junio. Así mismo, durante el período de estudio, entre el 03 al 04 de agosto del 2016, el paso del huracán Earl en el Caribe implicó un aumento en la precipitación en el área de estudio, coincidiendo con un mayor incremento en el radio del espejo de agua de las aguadas, especialmente para El Tintal y Los Monifatos (Instituto Nacional de Sismología, Vulcanología, Meteorología e Hidrología [Insivumeh], 2016). Lo anterior, evidencia que fenómenos meteorológicos estocásticos intensos, como los huracanes, tienen una influencia significativa en el régimen de acumulación de agua en los cuerpos de agua, siendo también el caso de las aguadas (Hanes \& Stromberg, 1998).

Además de los patrones de acumulación del agua interceptada por las aguadas, las variaciones físicas y bióticas determinan que esta agua se pierda como escorrentía o evapotranspiración. La topografía también influye si hay áreas de captación más extensas y podrían prolongar la duración de la fase de inundación y acortar la fase de desecación (Keeley \& Zedler, 1998). Este podría ser el caso de El Tintal, el cual se encuentra en un bajo o selva inundable y la acumulación de agua podría depender del flujo de la misma desde áreas con relieve más elevado, a lo cual se podrían deber las pequeñas diferencias que se observaron. Se desconoce además, la influencia de la permeabilidad del sustrato sobre el balance hídrico de cada aguada, donde suelos con niveles más altos de arcillas pueden favorecer la acumulación de agua (Bauder, 2005; Keeley \& Zedler, 1998; Wahl, Schreiner, Byrne, \& Hansen, 2007).

\section{Registro de variables microclimáticas}

Respecto a la evapotranspiración, la magnitud de la misma se relaciona con factores físicos a escala local, como la temperatura, humedad y viento. La vegetación circundante modifica además, la incidencia solar y el flujo de aire que afectan la evaporación, por lo tanto, las diferencias en la composición estructural de la vegetación de las aguadas podría explicar las diferencias en el volumen de agua acumulada (Mitsch \& Gosselink, 2015). Por ejemplo, El Trampole y El Tintal presentaron mayores valores de temperatura y menores valores de humedad relativa, y bajos valores para el radio de la aguada, lo que indica una mayor tasa de evapotranspiración. Siendo estas aguadas ubicadas en bajos, donde los árboles mayores a $30 \mathrm{~m}$ se encuentran ausentes y la cobertura herbácea es densa, con vegetación susceptible a inundaciones, lo cual pudo influir en una mayor exposición a la radiación solar y transpiración por la vegetación herbácea (González, 2015; Lundell, 1937; Rodas, 1998). De forma contraria, la Poza Maya se encuentra en un bosque alto con mayor cobertura del estrato arbóreo, lo que podría determinar que presente mayores valores de volumen de agua (González, 2015). Es importante hacer notar que en el estudio de González (2015) se describen como aguadas permanentes al menos a tres de las aguadas incluidas en este estudio, las cuales se secaron en el 2016. Esto evidencia que los patrones de disponibilidad de agua en las aguadas varían en cada año, dependiendo de los patrones de precipitación, duración e intensidad de las lluvias (Bauder, 2005).

Todos estos cambios estacionales en el régimen de las lluvias, en el clima y la humedad relativa, ocasionan variaciones del balance hídrico de las aguadas, que determina a su vez, la acumulación de agua o la pérdida de la misma. Patrones que pueden relacionarse además con el establecimiento de la vegetación. Zedler (1987) caracterizó al menos cuatro estadios en las pozas estacionales en California en un año, iniciando con la germinación de semillas latentes y el crecimiento de plantas perennes, seguida de una fase acuática o inundable, para después disminuir el nivel del agua, 
finalizando con una fase de sequía. En este estudio, todas las aguadas presentaron fases inundables, en concordancia con la precipitación, y una fase de sequía, acompañados por una sucesión de vegetación asociada. En los registros fotográficos pudo observarse la presencia de plantas acuáticas en las aguadas, donde las aguadas Monifatos, El Tintal y Poza Maya presentaron una cobertura de hidrófitas emergentes y flotantes, que podrían influir en la velocidad con la que el agua acumulada se evapotranspira, fluctuación particularmente evidente en la Poza Maya. Por lo tanto, puede inferirse que se lleva a cabo una sucesión de distintos grupos funcionales y taxonómicos que aprovechan los recursos que ofrece los patrones de humedad y productividad asociados al hidroperíodo de cada aguada, como ya se ha reportado en la literatura (Husband \& Barrett, 1998; Jeffries, 2001; Schriever, Cadotte, \& Wililams, 2014).

El estudio de cuerpos de agua fluctuantes como las aguadas es complejo, ya que las medidas sobre el tamaño y forma son representativas únicamente para un momento en particular, debido a que llegan a cambiar a lo largo del tiempo, incluso llegando varios cuerpos de agua a unirse o bien, a secarse (Zedler, 1987). Siendo otra de las variables que determina la permanencia del agua el aislamiento del subsuelo y la geomorfología de la aguada (Akpinar, 2011). En este sentido, se considera importante realizar el monitoreo de los niveles del agua a través de varios años e investigaciones que incorporen estudios topográficos de las aguadas. La comprensión de la variación geomorfológica de las aguadas y su influencia en la distribución y balance de agua, puede ayudar a predecir qué aguadas serán clave, tanto a nivel de área protegida como de paisaje en la hidratación de la fauna presente, o bien, cuáles aguadas son susceptibles a un manejo especial para evitar su desecamiento.

\section{Correlación entre los datos de temperatura y humedad relativa en aguadas y la EMA Ca- lakmul II}

Con respecto a la correlación significativa entre los valores registrados por los sensores en las aguadas y la EMA Calakmul II, indica que es posible utilizar los valores registrados por la EMA como una referencia de la temperatura y humedad relativa de las aguadas en los programas de monitoreo de aguadas y la fauna asociada. Sin embargo, dadas las variaciones climáticas interanuales, se recomienda implementar un estudio de varios años, con el fin de determinar si efectivamente se mantiene esta correlación significativa a través de años con distintas características climáticas.
En cuando a las recomendaciones para el manejo de las aguadas, es evidente que en años como el 2016, en el cual se secan por completo las aguadas, podría suponerse la necesidad de implementar medidas alternativas como los bebederos artificiales que se han instalado en la RBC (Araujo, 2014). Sin embargo se considera que, con base al principio de precaución, es necesario realizar estudios sobre la calidad de agua en estas fuentes alternativas, con el fin de garantizar que no representan ningún riesgo para la fauna silvestre que las utilice.

Así mismo, podría ser que la fauna esté adaptada para estas condiciones, migrando hacia otras áreas, como el caso del indidivuo de T. bairdii que reportan Reyna-Hurtado, Sanvicente-López, Pérez-Flores, Carrillo-Reyna y Calmé (2016) en la RBC, el cual se desplazó una distancia máxima de $10.5 \mathrm{~km}$, aparentemente en busca de agua. Por lo que otra estrategia a considerar es mantener la conectividad entre las aguadas para permitir el desplazamiento de la fauna, sin necesidad de otras medidas alternativas, cuyo costo de implementación podría hacerlas inviables (Araujo, 2014; O'Farrill et al., 2014). Sin embargo, un riesgo asociado a estas potenciales migraciones, es que las especies se vean forzadas a salir afuera de las áreas protegidas hacia zonas pobladas donde aumenta su vulnerabilidad a la cacería o atropellamientos (O'Farrill et al., 2014).

De modo que se plantea la necesidad del desarrollo de estrategias integrales a escala de paisaje, que aseguren el movimiento de las especies, acciones de control y vigilancia para reducir la cacería, la conservación de los cuerpos de agua, medidas de mitigación en carreteras y educación ambiental en comunidades aledañas y la población en general (Araujo, 2014; González, 2015; O'Farrill et al., 2014).

\section{Agradecimientos}

La realización de este estudio fue posible gracias al cofinanciamiento de la Digi-Usac (2016), proyecto (4.8.63.4.04) y el Programa Mundial para la Conservación de los Tapires del Grupo de Especialistas del Tapir de la UICN y la Fondation Segré. Así como la participación del personal de Cecon Usac: Saúl Castillo Hernández, Darwin Aguirre Castillo, Jaime F. Gutiérrez, Héctor A. Palacios, Agustín Caal Cajbón, Elvis Solís Montúfar, Erwin R. Mayen, Marvin A. Ochaeta, Renan Sotó Hernández, Fredy N. Bedoya y los técnicos Marvin Rosales, Juan J. Romero y Luis Rodas, la Fundación Defensores de la Naturaleza y los colabora- 
dores Raquel Leonardo y Santiago Zetina. Al Servicio Meteorológico Nacional de la Comisión Nacional del Agua (Conagua) de México por compartir información correspondiente a la estación meteorológica automática (EMA) Calakmull II. Y al Consejo Nacional de Áreas Protegidas (Conap) por su aval, acompañamiento y apoyo en la realización de este estudio.

\section{Referencias}

Akpinar, E. (2011). Aguadas: A significant aspect of the southern Maya lowlands water management system (Tesis de doctorado). University of Cincinnati, Ohio.

Anderson, E. R., Cherrington, E. A., Flores, A. I., Pérez, J. B., Carrillo, R., \& Sempris, E. (2008). Potential impacts of climate change on biodiversity in Central America, Mexico and Dominican Republic. Panamá: Centro del Agua del Trópico Húmedo para América Latina y el Caribe y United States Agency for International Development.

Araujo, R. (Ed.) (2014). Programa municipal de desarrollo de gran visión para el municipio de Calakmul, Campeche (2013-2040). Guatemala: Deutsche Gesellschaft für Internationale Zusammenarbeit.

Bauder, E. (2005). The effects of an unpredictable precipitation regime on vernal pool hydrology. Freshwater Biology, 50(12), 2129-2135. doi:10.1111/ j.1365-2427.2005.01471.x

Brooks, R. T. (2004). Weather-related effects on woodland vernal pool hydrology and hydroperiod. Wetlands, 24(1), 104-114. doi:10.1672/0277-5212(2 004)024[0104:WEOWVP]2.0.CO;2

Consejo Nacional de Áreas Protegidas, Dirección General del Patrimonio Cultural y Natural, \& Centro de Estudios Conservacionistas. (2010). Plan Maestro 2009-2013 Parque Nacional Mirador Río Azul y Biotopo Protegido Naachtún-Dos Lagunas. Guatemala: Autor.

Consejo Nacional de Áreas Protegidas. (2015). Plan Maestro de la Reserva de la Biósfera Maya. Segunda Actualización (Tomo 1). Guatemala: Autor.

González, V. R. (2015). Vertebrados medianos y mayores asociados a las aguadas del Biotopo Protegido Naachtún - Dos Lagunas, Petén (Tesis de licenciatura). Facultad de Ciencias Químicas y
Farmacia, Universidad de San Carlos de Guatemala, Guatemala.

Hanes, T., \& Stromberg, L. (1998). Hydrology of vernal pools on non-volcanic soils in the Sacramento Valley. En C. Witham, E. Bauder, D. Belk, W. Ferren \& R. Ornduff (Eds.), Ecology, Conservation, and Management of Vernal Pool Ecosystems. Proceedings from a 1996 Conference of the California Native Plants Society. Sacramento: California, Native Plant Society.

Hansen, M. C., Potapov, P. V., Moore, R., Hancher, M., Turubanova, S. A., Thau, D., ... Townshend, J. R. G. (2013). High-resolution global maps of 21 st-century forest cover change. Science, 342(6160), 850-853. doi:10.1126/science. 1244693

Hodgon, B. D., Hughell, D., Ramos, V. H., \& McNab, R. B. (2015). Tendencias en la deforestación de la Reserva de Biosfera Maya, Guatemala 20002013. Guatemala: Rainforest Alliance, Wildlife Conservation Society y Consejo Nacional de Áreas Protegidas.

Husband, B. C., \& Barrett, S. C. H. (1998). Spatial and temporal variation in population size of $\mathrm{Ei}$ chhornia paniculata in ephemeral habitats: Implications for metapopulation dynamics. Journal of Ecology, 86(6), 1021-1031. doi:10.1046/j.13652745.1998.00324.x

Instituto Nacional de Ecología. (2000). Programa de manejo de la Reserva de Biosfera Calakmul. México: Autor.

Instituto Nacional de Sismología, Vulcanología, Meteorología e Hidrología. (2016). Avance de la Tormenta Earl. (Informe preliminar, emisión 4 agosto 2016). Recuperado de www.insivumeh. gob.gt/meteorologia/Informe_preliminar_tormenta_earl.pdf

Jeffries, M. J. (2001). Modeling the incidence of temporary pond microcrustacea: The importance of dry phase and linkage between ponds. Israel Journal of Zoology, 47(4), 445-458. doi:10.1560/8C99UNJK-V1CL-EA8K

Keeley, J., \& Zedler, P. (1998). Characterization and global distribution of vernal pools. En C. Witham, E. Bauder, D. Belk, W. Ferren \& R. Ornduff (Eds.), Ecology, Conservation, and Management 
of Vernal Pool Ecosystems. Proceedings from a 1996 Conference of the California Native Plants Society. Sacramento, CA Native Plant Society.

Leibowitz, S. G., \& Brooks, R. T. (2007). Hydrology and landscape connectivity of vernal pools. En A. Calhoun \& P. De Maynadier. Science and conservation of vernal pools in Northeastern North America, (p. 31-54). Nueva York: CRC Press.

Lundell, C. L. (1937). The Vegetation of Petén. Studies of Mexican and Central American Plants I. Washington, D. C.: Carnegie Institution of Washington.

Mitsch, W., \& Gosselink, J. (2015). Wetlands ( $5^{\text {th }}$ ed.). New Jersey: Wiley \& Sons

Moreira-Ramírez, J. F., Reyna-Hurtado, R., Hidalgo-Mihart, M., Naranjo, E., Ribeiro, M. C., García-Anleu, ... Ponce-Santizo, G. (2016). Importancia de las aguadas para el pecarí de labios blancos (Tayassu pecari) en la Selva Maya, Guatemala. Therya, 7(1), 51-64. doi: 10.12933/ therya-16-344.

O’Farrill, G., Schampaert, K. G., Rayfield, B., Bodin, O., Calmé, S., Sengupta, R., \& Gonzalez, A. (2014). The potential connectivity of waterhole networks and the effectiveness of a protected area under various drought scenarios. Plos One, 9(5), e95049. doi:10.1371/journal.pone.0095049

Pérez-Cortez, S., Enríquez, P. L., Sima-Panti, D., Reyna-Hurtado, R., \& Naranjo, E. J. (2012). Influencia de la disponibilidad de agua en la presencia y abundancia de Tapirus bairdii en la selva de Calakmul, Campeche, México. Revista Mexicana de Biodiversidad, 83(3), 753-761. doi:10.7550/ rmb.25095

Reyna, R., O'Farril, G., Simá, D., Andrade, M., Padilla, A., \& Sosa, L. (2010). Las aguadas de Calakmul: Reservorios de vida silvestre y de la riqueza natural de México. Biodiversitas, 93, 1-6.

Reyna-Hurtado, R., Sanvicente-López, M., Pérez-Flores, J., Carrillo-Reyna, N., \& Calmé, S. (2016). Insights into the multiannual home range of a Baird's tapir (Tapirus bairdii) in the Maya Forest. Therya, 7(2), 271-276. doi:10.12933/therya-16-348

Rodas, R. (1998). Evaluación de la riqueza de especies del dosel y del sotobosque en la estación biológi- ca las Guacamayas, Parque Nacional Laguna del Tigre, Petén (Tesis de licenciatura). Universidad de San Carlos de Guatemala, Facultad de Ciencias Químicas y Farmacia, Guatemala.

Ruano, G., Moreira, J., García, R., McNab, R. B., Ponce-Santizo, G., Méndez, V., \& Córdova, F. (2009). Monitoreo de manadas de jabalí y dantos que visitan las aguadas de la región Este del Parque Nacional Mirador Río Azul (Informe técnico). Guatemala: Wildlife Conservation Society, United States Agency for International Development, Consejo Nacional de Áreas Protegidas y Centro de Estudios Conservacionistas.

Sandoval-Serés, E., Reyna-Hurtado, R., Briceño-Méndez, M., \& de la Cerda-Vega, R. (2016). Uso de aguadas y abundancia relativa de Tapirus bairdii en la región de Calakmul, Campeche, México. Therya, 7(1), 39-50. doi: 10.12933/therya-16-349, ISSN 2007-3364

Schriever, T. A., Cadotte, M. W., \& Williams, D. D. (2014). How hydroperiod and species richness affect the balance of resource flows across aquatic-terrestrial habitats. Aquatic Sciences, 76(1), 131-143. doi: 10.1007/s00027-013-0320-9

Simá, P., Reyna, R, \& Retana, O. (2008). Caracterización de fauna silvestre asociada a aguadas en cuatro ampliaciones forestales en la Reserva de Biosfera Calakmul, Campeche, México (Informe técnico). México: Reserva de Biosfera Calakmul, ProNatura Península de Yucatán, The Nature Conservancy, Universidad Autónoma de Campeche y Universidad de Florida.

Wahl, D., Schreiner, T, Byrne, R., \& Hansen, R. (2007). A Paleoecological record from a late classic Maya Reservoir in the North Peten. Latin American Antiquity, 18(2), 212-222. doi:10.2307/25063105

Winter, T. C., Rosenberry, D. O., Buso, D., \& Merk, D.A. (2001). Water source to four U.S. wetlands: Implications for wetland management. Wetlands, 21(4), 462-473. doi:10.1672/0277-5212(2001)0 21[0462:WSTFUS]2.0.CO;2

Zedler, P. (1987). The ecology of southern California Vernal Pools: A community profile. Biological Report 85(7.11). Washington, D. C.: Fish and Wildlife Service. 